\title{
DUAL CLUSTERING IN VISION SYSTEMS FOR ROBOTS DEPLOYED FOR AGRICULTURAL PURPOSES
}

\author{
Alexander Tyryshkin ${ }^{\mathrm{a}}$, Alexander Belyaev \\ Tomsk State University, 634050, Tomsk, Russia
}

\begin{abstract}
Continuously variable parameters of environment of robots' functioning complicate their use in agriculture. Record of disturbing actions only by means of software leads to complication of the programs. In turn, this leads to rise in price of the software product and reduction of robot's operational reliability. The authors suggest carrying out preliminary adaptation of the vision system to environment by means of hardware. Hardware is selected automatically based on artificial intelligence.
\end{abstract}

\section{Introduction}

Rapid development of robotics requires creation of more and more sophisticated systems of sensitization. Machine vision is the most developed among artificial sensors. Modern robots can easily work in factory shops and public space. In these cases, the observed objects are in artificial environment - in a pre-determined illumination range with a known emission spectrum, in a limited range of humidity and dust level. Gained success has inspired developers for a wider use of robots in natural environment. At the same time, agriculture is almost a new area of application of robots.

\section{Factors, which complicate realization of agricultural robots}

Application of robots for handling and harvesting of seed grains is constrained by complexity of creation of control systems because of the following factors:

- Color gamma of cultivated plants is a little different from color gamma of weeds.

- Color gamma of cultivated plants changes with the growth and maturation.

- Image depends on the direction of light.

- Spectrum of solar radiation depends on the time of day and humidity.

- Image depends on the strength and direction of wind.

This is not a complete list of disturbing factors. The image also depends on the plant lodging, presence of fog, dew, dust, etc.

\footnotetext{
${ }^{a}$ Corresponding author:taw@ie.tusur.ru
} 
Figure 1 shows images obtained by the web-cam in different directions («towards the sun» and «opposite to the sun»). It is very problematic to recognize plants on the image b) even by the «natural» neural network.

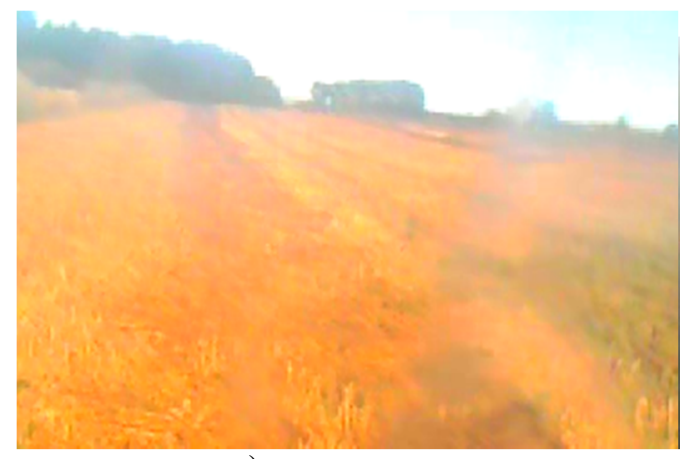

a)

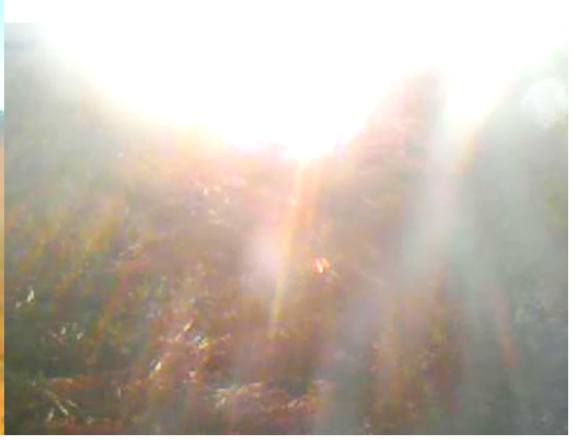

b)

Figure 1. a) The sun is behind; b) the sun is in front.

Figure 2 shows images of the same mown buckwheat. Figure 2, $a$ shows an image of buckwheat before threshing. As soon as threshing starts (Figure 2, b), dust appears, which «lights» the image in the sunrays.

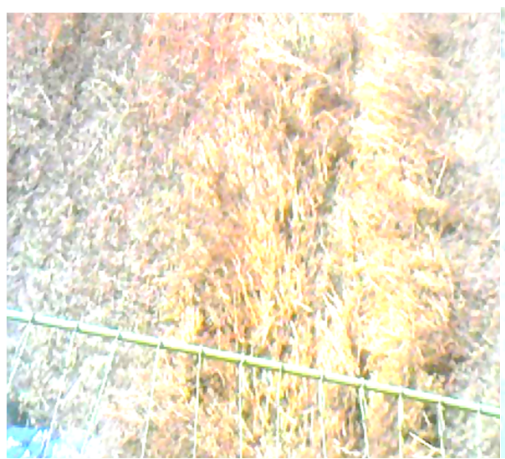

a)

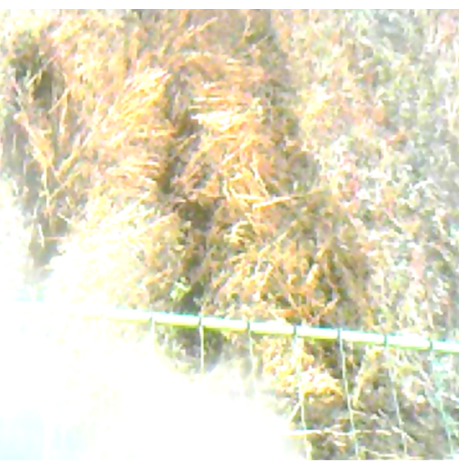

b)

Figure 2. Mown down buckwheat before threshing (a) and during a threshing process (b).

In addition to environmental and climatic factors, presence of weeds and diseases of cultivated plants complicate the task of image processing. On Figure 3 we can see dust because of such disease as gray rust.

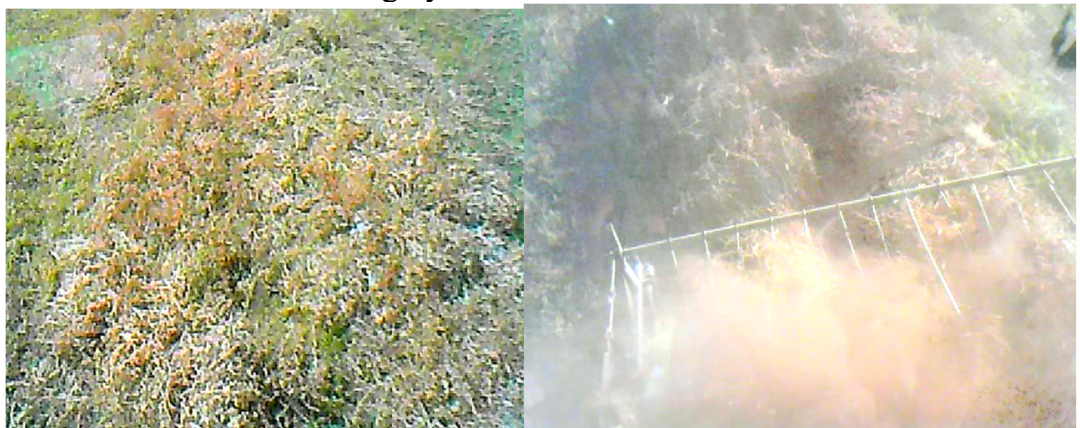

a)

Figure 3. Gray rust on peas. a) before threshing; b) during the harvesting process. 


\section{Existing approaches to solution of image recognition tasks}

Today, attempts to process images have been made by all known methods including both the simplest methods based on change of brightness, contrast, coloration, and the latest developments in the field of artificial intelligence [1]. In the simplest methods, image binarization is a key point. The ultimate goal of these methods is to divide objects of the working area into classes «necessary object» / «unnecessary object». For example, not mown areas/ mown areas would be such objects for agricultural machines. Simple methods do not work for recognition of such natural objects as cultivated plants and weeds. In some cases, fuzzy logic, genetic algorithms and neural networks give a satisfactory result $[2,3]$. However, classical neural networks require large computing resources, greater efforts for training and do not guarantee achievement of the required quality.

It is possible to reduce requirements to computing resources for image processing by use of convolutional neural networks [4]. However, high cost and lack of sufficient number of appropriate specialists in the manufacturing industry constrain introduction of sophisticated computer technology in agriculture.

According to the authors, development of robots for agricultural purposes should involve not only increase of control systems "intelligence" but also search of engineering solutions aimed to simplify tasks facing developers.

\section{Suggested approach to solution of the task}

Creation of local areas of "comfortable work" of vision systems is one of such solutions. It is necessary to make working conditions of Outdoor-type robots closer to working conditions of Indoor-type robots. There is no direct solution for this problem because it is impossible to extend technosphere to the entire wildlife. Consequently, it is necessary to create artificial conditions only in the robot's "field of interest". Moreover, the robot must automatically adapt the field of work in conditions of changing environmental parameters.

Figure 4 shows the proposed structure of the agricultural machine control system.

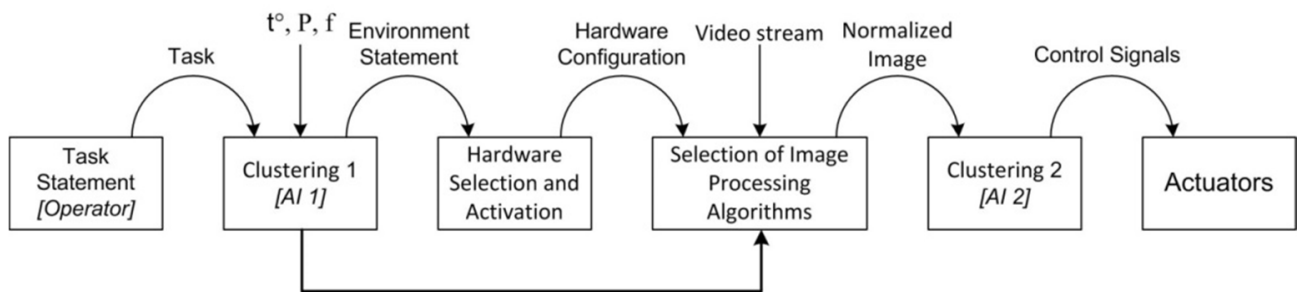

Figure 4. Structure of the control system.

Artificial intelligence (AI) is divided into two parts - AI1 and AI2. In addition to the task set by the operator, AI1receives information on the environment: temperature, pressure, humidity, light intensity, etc. Based on these data, AI1 determines one of the known typical situations. For example, fine, dew, twilight, fog. In fact, it is a task of clustering, which can be solved based on Kohonen maps [5]. This type of artificial intelligence does not require large computational resources. It is possible to realize Kohonen maps based on industrial controllers of general purpose. Training of Kohonen maps does not require highly skilled professionals. Results of training are intuitively understandable and easily testable.

Identification of situation is followed by automatic selection of hardware aimed at creating favorable conditions for operation of the artificial intelligence unit AI2. These can 
include sources of artificial lighting; hardware filters both of visible spectrum and of the infrared band; fans; windscreen wipers; and others.

After the work done, partially adapted image, called as Normalized, goes to the input of clustering unit 2.

Despite the complexity of optical image processing [6] for agricultural robot, the vision task itself is relatively simple. It is necessary either to determine mown plants (presence or centerline), or to determine the boundary between the tilled and untilled area. Thus, the image should be divided into two zones: non-working area / working area. Again, this is a relatively simple task of clustering, which can be solved by the clustering unit 2 .

After determining the boundary of the working area, control signals go to actuating components (ACs), which influence the units of agricultural machinery.

The proposed approach begins with the definition of range of tasks, which are to be solved by robots of this type. These tasks can include determining the boundaries of mown plants; selection of mown plants, briquetting and unloading on a given line; mowing of lodged plants, etc. Each of these tasks is independent and requires personal approach to its solution. As far as these tasks are solved, obtained solutions can be combined in a single software product to create control systems for universal agricultural machines.

The next step is creation of a base of model images, which are typical for a specific task. This work should be carried out during the growing season along with development of the robot hardware. The cost of this work is low because the vision system (VS) can be installed on existing agricultural machines commonly working in the fields. Such usage of VS will allow accumulating video data obtained in different environmental conditions: variable level of light, presence / absence of fog, etc.

The next stage of the work can be cameral. On this step, it is necessary to select the neural network topology and training it. Instrumental preparation for image obtaining (use of additional lighting, filters, etc.) should allow reducing the complex task of image analysis to relatively simple task of clustering.

After completing these works, it is possible to use the control system in test mode. In this case, an operator provides actual control of an agricultural machine and the control system acts as an expert system. The created system can be converted from the training mode to automatic control mode when during debugging solutions of the expert system become adequate to the operator's solutions.

\section{Acknowledgment}

The paper was written as part of the research project No. 8.2.31.2015, carried out with the support of the Program "Research Foundation of Tomsk State University named after D.I. Mendeleev" in 2015 - 2016, grant RFBR No. 16-29-04388/16.

\section{References}

[1] A.V. Tkachev, S.V. Shanygin, Young Scientist (19), 215 (2015)

[2] C. J. Lakhmi, N.M. Martin, Fusion of Neural Networks, Fuzzy Systems and Genetic Algorithms: Industrial Applications (CRC Press, United States ,1998)

[3] M.A. Ranzato, C.Poultney, S. Chopra and Y. LeCun, Advances in Neural Information Processing Systems, 1137 (2007)

[4] M. Zeiler, D. Krishnan, G. Taylor, R. Fergus, 2010 IEEE Conference on computer vision and pattern recognition (CVPR), $2528 \quad$ (2010) doi:10.1109/CVPR.2010.5539957 
[5] T. Kohonen, Self-Organizing Maps (Springer Science \& Business Media, New York, 2012)

[6] D.V. Shashev, S.V. Shidlovskiy, V.I. Syriamkin, A.V. Yurchenko, IOP Conference Series: Materials Science and Engineering 81, 012101 (2014) doi: 10.1088/1757899X/81/1/012101 